\title{
Octreotide Alleviates Autophagy by Up- Regulation of MicroRNA-101 in Intestinal Epithelial Cell Line Caco-2
}

\author{
Yuling $\mathrm{Li}^{\mathrm{a}}$ Su Wang ${ }^{\mathrm{b}}$ Xingjuan Gao ${ }^{c}$ Ying Zhao ${ }^{\mathrm{d}} \quad$ Yongwei $\mathrm{Li}^{\mathrm{e}}$ \\ Bin Yang ${ }^{f}$ Naili Zhang ${ }^{g}$ Lina Ma ${ }^{h}$
}

aDepartment of Pathophysiology, Binzhou Medical University, Yantai, 'bepartment of Cardio-Thoracic Surgery, The 305 Hospital of People's Liberation Army, Beijing, 'Department of Pediatrics, The Affiliated Yantai Yuhuangding Hospital of Qingdao University, Yantai, dDepartment of Intensive Care Unit, Shenzhen People's Hospital, Shenzhen, e'Department of Urology Surgery, The Affiliated Yantai Yuhuangding Hospital of Qingdao University, Yantai, fDepartment of Gynecology, The Affiliated Yantai Yuhuangding Hospital of Qingdao University, Yantai, 9Department of Anatomy, Binzhou Medical University, Yantai, hepartment of Diagnostics, Binzhou Medical University, Yantai, China

\section{Key Words}

Intestinal mucositis • Autophagy • miR-101 • TAK1 • AMPK/mTOR

\begin{abstract}
Background: Intestinal mucositis is a common side-effect after anti-cancer therapy, which may greatly restrict the therapeutic effects. We aimed to explore the functional role of octreotide (OCT) in lipopolysaccharide (LPS)-induced autophagy of human intestinal epithelial cells as well as the underlying mechanisms. Methods: Cell viability and expression of proteins related to autophagy, AMPK and the mTOR pathway in LPS-treated Caco-2 cells were determined by CCK-8 assay and Western blot analysis, respectively. Effects of OCT on LPS-induced alterations as well as miR-101 expression were measured. Then, miR-101 was aberrantly expressed, and whether OCT alleviated LPS-induced autophagy through miR-101 was tested. Next, whether TGF- $\beta$-activated kinase 1 (TAK1) was involved in the regulation of miR-101 in LPS-induced autophagy was studied. Effects of OCT on monolayer permeability and tight junction level were analyzed via measuring transepithelial electrical resistance (TEER) and expression of tight junction proteins. Results: LPS reduced cell viability and increased autophagy through activating AMPK and inhibiting the mTOR pathway in Caco-2 cells. OCT alleviated LPSinduced alterations and repressed degradation of autophagosome. Then, we found that OCT affected autophagy through up-regulating miR-101 in LPS-treated cells. Moreover, miR-101induced inactivation of AMPK and activation of the mTOR pathway in LPS-treated cells were reversed by inhibition of TAK1 phosphorylation. Finally, we found miR-101 was up-regulated
\end{abstract}

Y. Li, S. Wang and X. Gao contributed equally to this work.

\begin{tabular}{ll}
\hline Yuling Li & Department of Pathophysiology, Binzhou Medical University \\
& No. 346, Guanhai Road, Yantai 264003 (China) \\
E-Mail 122245errqif@gmail.com
\end{tabular}

\section{KARGER}


in differentiated cells, and OCT protected the monolayer permeability and tight junction level. Conclusion: OCT repressed autophagy through miR-101-mediated inactivation of TAK1, along with inactivation of AMPK and activation of the mTOR pathway in LPS-treated Caco-2 cells.

\section{Introduction}

Intestinal mucositis is a common, debilitating side-effect after chemotherapy or radiotherapy of cancer [1]. Clinical symptoms of intestinal mucositis include vomiting, anorexia, nausea, diarrhea and dyhydration [2]. Numerous factors are involved in the pathogenesis of intestinal mucositis, such as release of inflammatory cytokines, epithelial cell apoptosis and microbial colonization [3]. However, to our knowledge, there is no definitive therapy for intestinal mucositis, which greatly impacts the outcome of therapy because of the dose reductions and treatment discontinuation in tumor therapy.

Octreotide (OCT; amino acid sequence FCFWKTCT) is a synthetic, long-acting peptide analogue of naturally occurring hormone named somatostatin [4]. It is clinically utilized for treatments of acromegaly [5], advanced neuroendocrine tumors [6], vascular abnormalitiesinduced gastrointestinal bleeding [5], and liver cirrhosis-induced chylous ascites [7]. A previous study has reported that OCT ameliorates chemotherapy-induced diarrhea in patients [8], suggesting the potential application of OCT for alleviating intestinal mucositis after tumor therapy. Epithelial cells which are linked via tight junctions compose of intestinal barrier. Disruption of tight junctions as well as loss of intestinal barrier function is associated with intestinal mucositis [9]. Currently, somatostatin has been reported to protect intestinal barrier through regulating expression of tight junction proteins [10], verifying the protective role of OCT in intestinal mucositis. Molecular mechanisms on how the OCT affects intestinal cells are waiting to be revealed.

Autophagy is an evolutionary process, during which intracellular components are sequestered by autophagosomes, followed by degradation after fusion between autophagosomes and lysosomes [11]. Intestinal barrier dysfunction is accompanied by dysregulated autophagy in rats, showing that levels of LC3-II and Beclin-1 are increased in injured intestinal tissues [12]. Therefore, an association between autophagy and intestinal mucositis might be existed in intestinal cells. Considering the possible function of OCT on intestinal mucositis, we supposed that the functional role of OCT might be associated with autophagy. However, related literatures in intestinal epithelial cells are limited.

An increasing number of microRNAs (miRNAs/miRs; the small non-coding RNAs with a length of $\sim 22$ nucleotides) have been identified to participate in regulation of autophagy. For example, miR-17 and miR-30a are involved in autophagy by targeting different autophagypromoting genes in cancer cells $[13,14]$. Autophagy in human hepatocellular carcinoma cells was repressed by miR-101 [15]. Abnormal up-regulation of miR-101 might suppress autophagy in prostate cancer cells [16]. TGF- $\beta$-activated kinase 1 (TAK1) is a MAP kinase kinase kinase which can be activated by toll-like receptors (TLRs). Accumulating evidence has reported that TAK1 regulates phosphorylation of AMPK, and thereby represses the mTOR complex 1, leading to increase of autophagy [17]. Although the close relationship between miR-101 and autophagy as well as TAK1 and autophagy has been proven, the interaction among OCT, miR-101 and TAK1 remains unclear.

Intestinal mucositis is pathologically identified to be an intestinal inflammation [18]. Cell survival and induction of inflammatory cascades are crucial factors that influence the developments of intestinal mucositis [19]. Besides the immune response inductive effects, lipopolysaccharide (LPS) can also induce tight junction dysfunction, resulting in mucosal hyperpermeability [20]. Thus, in our study, we used LPS-induced Caco-2 cells to mimic in vitro intestinal mucosistis, and intended to figure out the effects of OCT on intestinal epithelial cell autophagy. The underlying molecular mechanisms possibly associated with miR-101 and TAK1 may provide innovative therapeutic targets for intestinal mucositis. 


\section{Cellular Physiology Cell Physiol Biochem 2018;49:1352-1363 \begin{tabular}{l|l|l} 
and Biochemistry Published online: 12 September, 2018 & $\begin{array}{l}\text { (c) } 2018 \text { The Author(s). Published by S. Karger AG, Basel } \\
\text { www.karger.com/cpb }\end{array}$ \\
\hline
\end{tabular}}

\section{Materials and Methods}

\section{Cell culture and treatment}

Human intestinal epithelial Caco-2 cells were obtained from American Type Culture Collection (ATCC; Manassas, VA, USA). Cells (passages 22-28) were maintained in high glucose Dulbecco's modified eagle's medium (DMEM; Gibco, Bethesda, MD, USA) supplemented with 20\% fetal bovine serum (FBS; Gibco) under standard conditions $\left(37^{\circ} \mathrm{C}\right.$, humidified atmosphere with $5 \% \mathrm{CO}_{2}$ and $95 \%$ air). To lower the effects of FBS on cell growth and protection against LPS treatment as well as support cell survival, for LPS treatment, cells were incubated in DMEM containing 0.5\% FBS and $0.1 \mu \mathrm{g} / \mathrm{mL}$ LPS (Sigma-Aldrich, St. Louis, MO, USA) for 24 $\mathrm{h}$ as described previously [21]. In OCT-treated groups, cells were pre-treated with $10 \mu \mathrm{M}$ OCT (Selleckchem, Munich, Germany) for $24 \mathrm{~h}$ prior to LPS treatments [22]. For stimulation with Bafiomycin A1 (Baf-A1), cells were incubated in DMEM containing $0.5 \%$ FBS and $10 \mathrm{nM}$ Baf-A1 (Sigma-Aldrich) for $24 \mathrm{~h}$. For inhibition of TAK1, (5Z)-7-oxozeaenol ( $0.5 \mu \mathrm{M}$, Sigma-Aldrich) was added into culture and the cells were pre-incubated for $6 \mathrm{~h}$.

\section{Cell viability assay}

Cell viability of treated Caco-2 cells was measured using a Cell Counting Kit-8 (CCK-8; Dojindo Molecular Technologies, Gaithersburg, MD, USA). As recommended by the manufacturer, cells were seeded into 96well plates with $5 \times 10^{3}$ cells/well and were grown at $37^{\circ} \mathrm{C}$ overnight. Then, cells were stimulated with LPS or/and OCT for indicated times, followed by addition of $10 \mu \mathrm{L}$ CCK-8 solution. Cells were then incubated for additional $1 \mathrm{~h}$ and the absorbance at $450 \mathrm{~nm}$ was read by a Microplate Reader (Bio-Rad, Hercules, CA, USA).

\section{Cell transfection}

miR-101 mimic, inhibitor and negative control (miR-NC) were synthesized by GenePharma Co. (Shanghai, China). Caco-2 cells were seeded into 24 -well plates at a density of $3 \times 10^{3}$ cells/well. When reached $70 \%-80 \%$ confluence, cells were transfected with miR-101 mimic or inhibitor, respectively, by using Lipofectamine 3000 reagent (Invitrogen, Carlsbad, CA, USA) on the basis of manufacturer's instructions. miR-NC was transfected into cells acting as control for transfected cells.

\section{Alkaline phosphatase (ALP) activity assay}

Differentiation of Caco- 2 cells was induced by additional incubation for 30 days after $100 \%$ confluence. The Day 0 for differentiation was defined as the time that cells reached $100 \%$ confluence. At Day 0, 2, 4, 7, 14 and 30 post-confluency, cells were collected and the ALP activity was determined as described previously [23]. The p-nitrophenylphosphate was used as an ALP substrate, and the reaction mixture was measured at $405 \mathrm{~nm}$. Relative ALP activity of cells at Day 2-30 was normalized to the ALP activity of cells at Day 0 .

\section{Measurements of transepithelial electrical resistance (TEER)}

Caco-2 cells were seeded into the upper chamber of 6-well Transwell plates (Corning, Corning, NY, USA) at a density of $1 \times 10^{5}$ cells/well. The Day 0 for differentiation was defined as the time that cells reached $100 \%$ confluence. After treatments, TEER was determined by using an EVOM $^{2}$ Epithelial Voltohmeter (World Precision Instruments, Sarasota, FL, USA), according to the manufacturer's instructions. The resistance value $(\mathrm{Ohm})$ of the blank (culture insert with culture medium) was subtracted from the resistance value of sample, and the final unit area resistance $\left(\mathrm{Ohm} \times \mathrm{cm}^{2}\right)$ was calculated by multiplying the sample resistance by the area of the membrane.

\section{Quantitative reverse transcription PCR ( $q R T-P C R)$}

Caco-2 cells were seeded into 6-well plates at a density of $1 \times 10^{6}$ cells/well. After treatments, total RNAs were extracted by using RNeasy mini kit (Qiagen, Valencia, CA, USA) following the supplier's protocol. Then, 500 ng RNA quantified by a Nanodrop 2000 system was reverse transcribed to cDNA using the Taqman MicroRNA Reverse Transcription Kit (Applied Biosystems, Foster City, CA, USA) or the PrimeScript ${ }^{\text {TM }}$ 1st Strand cDNA Synthesis kit (TaKaRa, Dalian, China). Real-time PCR for quantification of miR-101 and sucrase-isomaltase mRNA was performed using the Taqman Universal Master Mix II (Applied Biosystems) and the SYBR ${ }^{\circledast}$ Advantage ${ }^{\circledast}$ qPCR Premix (TaKaRa), respectively. Relative expression fold was determined 


\section{Cellular Physiology Cell Physiol Biochem 2018;49:1352-1363 and Biochemistry Published online: 12 September, $2018 \begin{aligned} & \text { DOI: } 2018 \text { The Author(s). Published by S. Karger AG, Basel } \\ & \text { www.karger.com/cpb }\end{aligned}$ \\ Li et al.: Role of Octreotide in Caco-2 Cells}

according to the $2^{-\Delta \Delta C t}$ method [24]. U6 and GAPDH were acted as the housekeeping genes for miR-101 and sucrase-isomaltase $\mathrm{mRNA}$, respectively.

\section{Western blot analysis}

Caco-2 cells were seeded into 6-well plates at a density of $1 \times 10^{6}$ cells $/$ well. After treatments, cells were harvested in ice-cold PBS, and lysed in RIPA lysis buffer containing $0.2 \mathrm{mM}$ PMSF and cocktail inhibitors of protease and phosphatases (all Beyotime, Shanghai, China). Then, concentration of proteins in the supernatants of the whole cell lysates was quantified using the BCA ${ }^{\text {TM }}$ Protein Assay Kit (Pierce, Appleton, WI, USA), and the protein samples were separated by SDS-PAGE. After that, proteins in the gels were transferred to the nitrocellulose membranes, followed by blocking of non-specific binding sites in the membranes with $5 \%$ fat-free milk. Those membranes were successively incubated with primary antibodies at $4^{\circ} \mathrm{C}$ overnight and horseradish peroxidase-labeled secondary antibody (goat anti-rabbit, ab205718, Abcam, Cambridge, UK) at room temperature for $1 \mathrm{~h}$. Primary antibodies include antibodies against microtubule-associated protein 1 light chain 3B (LC3B; ab48394), Beclin-1 (ab62557), p62/sequestosome 1 (p62; ab207305), claudin-1 (ab180158), zonula occludens-1 (ZO-1; ab96587), occludin (ab167161), $\beta$-actin (ab8227, all Abcam), total AMP-activated protein kinase (t-AMPK; 2532), phospho (p)-AMPK (2535), total mechanistic target of rapamycin (t-mTOR; 2972), p-mTOR (2971), total $70 \mathrm{kDa}$ ribosomal S6 kinase (t-p70S6K; 9208), p-p70S6K (9202), total TAK1 (t-TAK1; 4505) or p-TAK1 (4536, all Cell Signaling Technology, Beverly, MA, USA). Blots were visualized using an enhanced chemiluminescence kit (GE Healthcare Bio-Sciences, Pittsburgh, PA, USA). Intensity of the bands was quantified using the ImageJ software (version: 1.4.3.67; National Institutes of Health, Bethesda, MA, USA).

\section{Statistical analysis}

All experiments were repeated three times. Results in each figure were presented as the mean \pm standard error of the mean (SEM). Statistical analysis was performed using Graphpad Prism 5 software (GraphPad, San Diego, CA, USA). The $P$-values were calculated using the one-way analysis of variance (ANOVA) with Tukey's correction or multiple $t$-tests. Comparison with a $P$ value of $<0.05$ was considered as a significant difference.

\section{Results}

LPS reduced cell viability, and induced autophagy, activation of AMPK and inhibition of the mTOR pathway in Caco-2 cells

Caco-2 cells were stimulated with LPS to simulate intestinal mucositis in vitro. Compared with the control group, cell viability was significantly reduced by LPS treatments $(P<0.05$, Fig. 1A). Then, expression of key proteins involved in the autophagy was measured, and Western blot analysis revealed that expression of LC3B-II and Beclin-1 was dramatically increased $(P<0.01$ or $P<0.001)$ while expression of p62 was markedly decreased $(P<0.01)$ by LPS treatments as compared to the control group (Fig. 1B). Subsequent results showed phosphorylation of AMPK was observably elevated $(P<0.05$, Fig. $1 \mathrm{C})$ while phosphorylation of mTOR and p70S6K was dramatically decreased (both $P<0.01$, Fig. 1D) by LPS treatments compared with the control group. Results illustrated that LPS could decrease cell viability, and induce autophagy, AMPK activation and inhibition of the mTOR pathway in Caco- 2 cells.

\section{OCT attenuated LPS-induced alterations of Caco-2 cells}

To assess the effect of OCT on LPS-treated Caco-2 cells, cells were treated with LPS or LPS plus OCT. Non-treated cells were acted as control. Results showed LPS-induced alterations were notably mitigated by OCT, showing significant increase of cell viability $(P<0.05$, Fig. 2A), down-regulation of LC3B-II, Beclin- $1(P<0.01$ or $P<0.001$, Fig. $2 \mathrm{~B})$ and $\mathrm{p} / \mathrm{t}-\mathrm{AMPK}(P$ $<0.05$, Fig. 2C), and up-regulation of p62 ( $P<0.01$, Fig. 2B), p/t-mTOR and p/t-p70S6K $(P<$ 0.01 or $P<0.001$, Fig. 2D), when compared to the LPS group. Moreover, we also explored how OCT affected autophagy in Caco-2 cells using Baf-A1. Results in Fig. 2E showed expression of LC3B-II in the Control group was lower than the Baf-A1 group, and LC3B-II levels in groups 


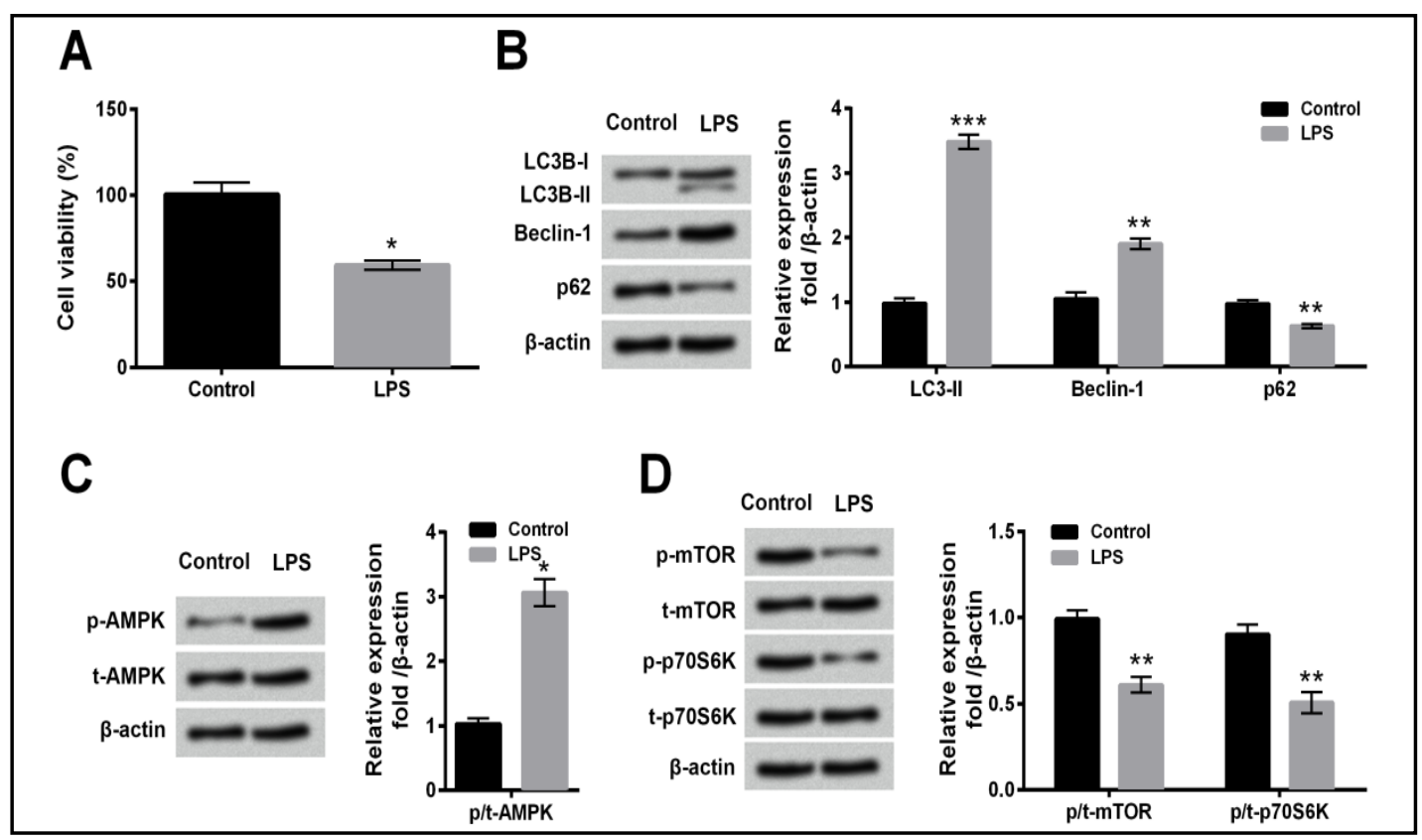

Fig. 1. LPS reduced cell viability, and induced autophagy, activation of AMPK and inhibition of the mTOR pathway. Caco- 2 cells were treated with or without $0.1 \mu \mathrm{g} / \mathrm{mL}$ LPS. A. Cell viability by CCK-8 assay. Expression of key proteins involved in autophagy (B), AMPK activation (C) and the mTOR pathway (D) was assessed by Western blot analysis. Data are presented as the mean \pm SEM of three independent experiments. ${ }^{*}, \mathrm{P}<0.05$; **, $\mathrm{P}<0.01$; ***, $\mathrm{P}<0.001$. LPS, lipopolysaccharide; AMPK, AMP-activated protein kinase; mTOR, mechanistic target of rapamycin; LC3B, microtubule-associated protein 1 light chain 3B; p62, p62/sequestosome 1; p70S6K, $70 \mathrm{kDa}$ ribosomal S6 kinase.

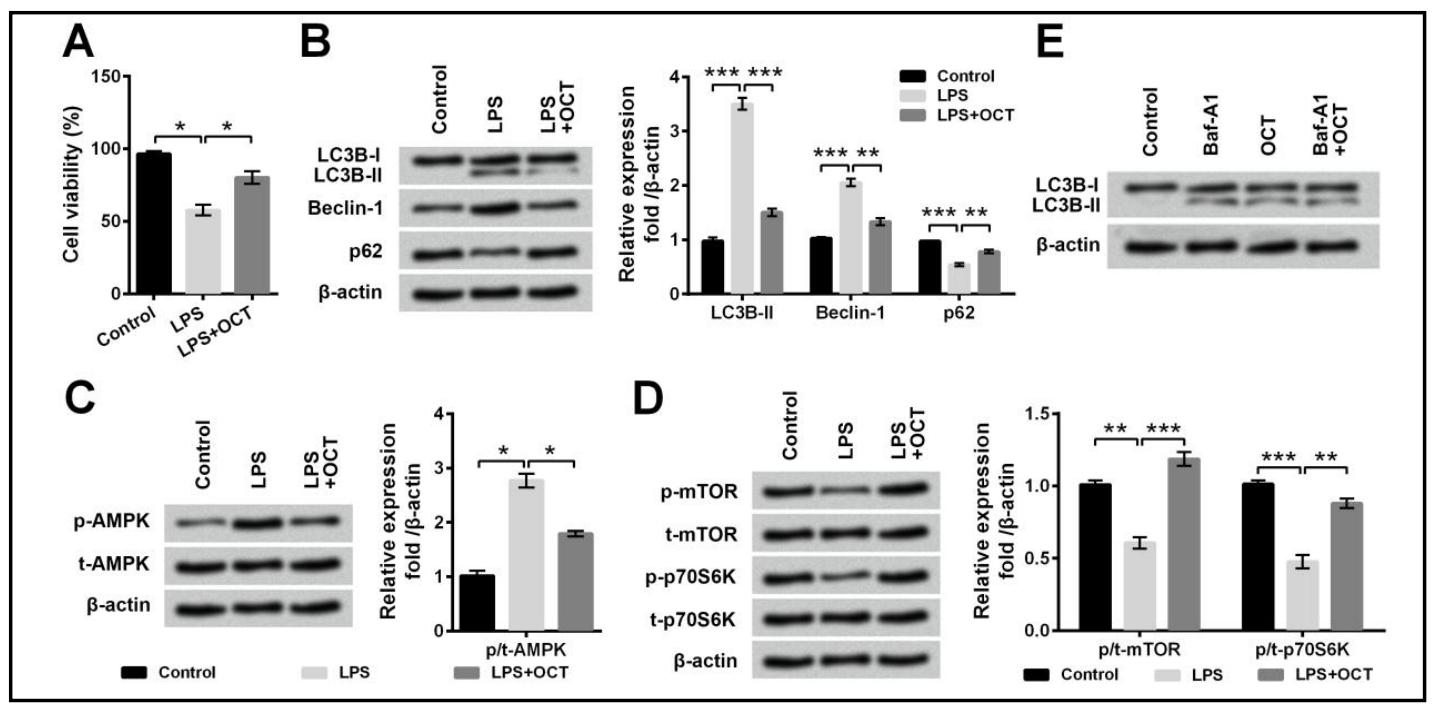

Fig. 2. LPS-induced alterations in Caco- 2 cells were attenuated by octreotide (OCT). Caco-2 cells were treated with $0.1 \mu \mathrm{g} / \mathrm{mL}$ LPS or LPS plus OCT $(10 \mu \mathrm{M})$. Non-treated cells were acted as control. A. Cell viability by CCK-8 assay. Expression of key proteins involved in autophagy (B), AMPK activation (C) and the mTOR pathway (D) was assessed by Western blot analysis. Caco-2 cells were treated with Bafiomycin A1 (Baf-A1; $10 \mathrm{nM}$ ) and/or OCT $(10 \mu \mathrm{M})$, and non-treated cells were acted as control. (E) Expression of LC3B was assessed by Western blot analysis. Data are presented as the mean \pm SEM of three independent experiments. *, $\mathrm{P}<0.05$; **, $\mathrm{P}<0.01$; ${ }^{* * *}, \mathrm{P}<0.001$. LPS, lipopolysaccharide; AMPK, AMP-activated protein kinase; mTOR, mechanistic target of rapamycin; LC3B, microtubule-associated protein 1 light chain 3B; p62, p62/sequestosome 1; p70S6K, $70 \mathrm{kDa}$ ribosomal S6 kinase. 
except the control group were equal, indicating that OCT might affect autophagy by repressing degradation of autophagosome. Those results indicated that OCT could mitigate LPS-induced alterations in Caco-2 cells.

\section{OCT up-regulated miR- 101}

The expression levels of miR-101 in Caco-2 cells stimulated with LPS or LPS plus OCT were measured. Non-treated cells were acted as control. As shown in Fig. 3, miR-101 level was nearly unchanged after LPS treatments compared with the control group. Interestingly, after treatments with OCT, miR101 level was significantly up-regulated as compared to the LPS group $(P<0.05)$. We thus concluded that OCT could up-regulate miR-101 in LPS-treated Caco-2 cells.

\section{OCT reduced Caco-2 cell} autophagy through upregulating miR-101

Following experiments were performed to explore whether OCT affected LPS-treated cells through up-regulating miR-101. Accordingly, miRs were transfected into Caco-2
Fig. 3. Expression of miR101 was up-regulated after octreotide (OCT) treatments in LPS-treated Caco-2 cells. miR-101 level was determined by quantitative reverse transcription PCR. Data are presented as the mean \pm SEM of three independent

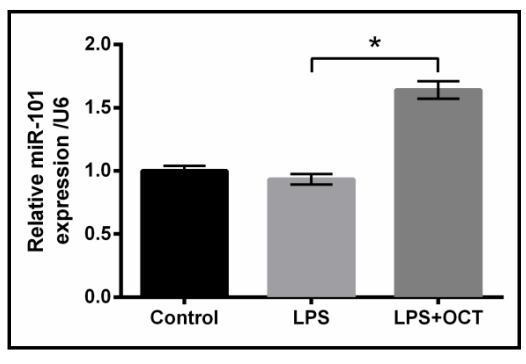
experiments. *, $\mathrm{P}<0.05$. LPS, lipopolysaccharide.

Fig. 4. Octreotide (OCT) repressed autophagy through up-regulating miR-101. Caco-2 cells were transfected with miR-101 mimic, inhibitor or negative control (miRNC). A. Expression of miR-101 by quantitative reverse transcription PCR. Transfected and nontransfected cells were incubated with $0.1 \mu \mathrm{g} /$ mL LPS or LPS plus OCT $(10 \mu \mathrm{M})$. Non-treated cells were acted as control. B. Expression of key proteins involved in autophagy by Western blot analysis. Data are presented as the mean \pm SEM of three independent experiments. ${ }^{* *}, \mathrm{P}<0.01$; $* * * \quad \mathrm{P}<0.001 . \quad$ LPS, lipopolysaccharide; LC3B, microtubule-associated protein 1 light chain $3 \mathrm{~B}$; p62, p62/sequestosome 1.

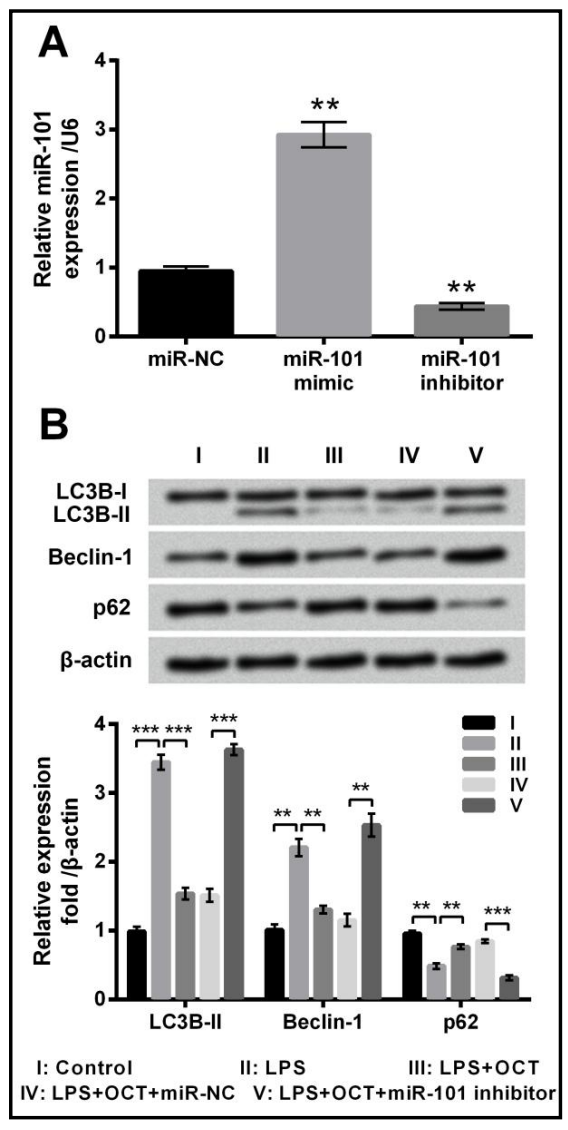

cells, and results in Fig. 4A showed miR-101 level in miR-101 mimic-transfected cells was prominently up-regulated while that in miR-101 inhibitor-transfected cells was significantly down-regulated as compared to the miR-NC group (both $P<0.01$ ). Western blot results in Fig. 4B showed that OCT-induced alterations of key proteins involved in autophagy were all dramatically reversed by miR-101 inhibition when compared to the LPS + OCT + miR-NC group $(P<0.01$ or $P<0.001)$. Data suggested that OCT might affect LPS-treated Caco- 2 cells through up-regulating miR-101.

Phosphorylation of TAK1 was decreased by miR-101

The phosphorylation of TAK1 in Caco-2 cells transfected with miRs was assessed. As evidenced by Fig. 5, phosphorylated level of TAK1 was remarkably reduced by miR-101 overexpression but was enhanced by miR-101 inhibition compared with the miR-NC group (both $P<0.05$ ). Results indicated that phosphorylation of TAK1 was negatively regulated by miR-101 expression in Caco-2 cells. 


\section{Cellular Physiology Cell Physiol Biochem 2018;49:1352-1363

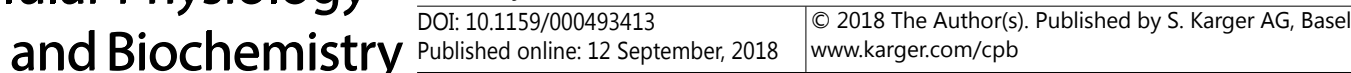 Li et al.: Role of Octreotide in Caco-2 Cells}

miR-101 inhibited AMPK phosphorylation and activated the mTOR pathway through inhibiting TAK1

We explored whether miR-101 affected LPStreated Caco-2 cells through TAK1. In Fig. 6A, LPS treatments significantly elevated phosphorylation of TAK1, and the elevation was reversed by miR-101 overexpression while was further increased by miR-101 inhibition (all $P<0.05$ ). In addition, phosphorylation of TAK1 was notably reduced after stimulation of (5Z)-7-oxozeaenol (TAK1 inhibitor) as compared to the LPS + miR-101 inhibitor group $(P<0.01)$. Further experiments showed, when compared to the LPS + miR-101 inhibitor group, miR-101 inhibition-induced increase of p/t-AMPK (Fig. 6B) as well as decreases of $\mathrm{p} / \mathrm{t}$-mTOR and p/t-p70S6K (Fig. 6C) was dramatically reversed by TAK1 inhibition $(P<0.01)$. Those results illustrated that miR-101 inactivated AMPK and activated the mTOR pathway through inactivating TAK1 in LPS-treated Caco-2 cells.

miR-101 was up-regulated in differentiated cells and OCT protected the monolayer permeability and tight junction level

Finally, the effects of miR-101 and OCT on differentiated Caco-2 cells were investigated. In Fig. 7A-7B, the significant increases of ALP activity and sucrase-isomaltase expression $(P<0.05, P<0.01$ or

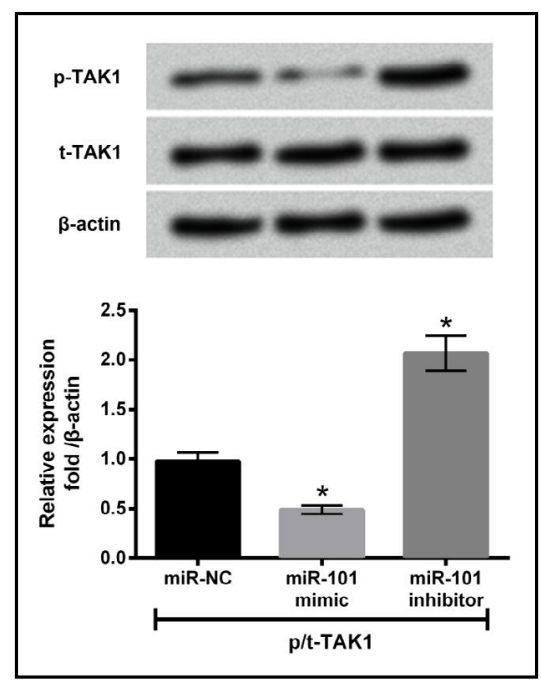

Fig. 5. Phosphorylation of TGF- $\beta$ activated kinase 1 (TAK1) was reduced by miR-101 overexpression. Caco-2 cells were transfected with miR-101 mimic, inhibitor or negative control (miR-NC). Phosphorylation of TAK1 was evaluated by Western blot analysis. Data are presented as the mean \pm SEM of three independent experiments. *, $\mathrm{P}<0.05$.
Fig. 6. miR-101 inactivated AMPK and activated the mTOR pathway through repressing TAK1 phosphorylation in LPStreatedCaco- 2 cells. Transfected and non-transfected cells were incubated with $0.1 \mu \mathrm{g} / \mathrm{mL}$ LPS or LPS plus (5Z)-7-oxozeaenol (0.5 $\mu \mathrm{M}$; TAK1 inhibitor). Nontreated cells were acted as control. Phosphorylation of TAK1 (A), AMPK (B), and key kinases in the mTOR pathway (C) was assessed by Western blotanalysis. Dataare presented as the mean \pm SEM of three independent experiments. * $\quad \mathrm{P}<0.05$; **, $\mathrm{P}<0.01$. LPS, lipopolysaccharide; TAK1, TGF$\beta$-activated kinase 1 ; AMPK, AMP-activated protein kinase; mTOR, mechanistic target of rapamycin.
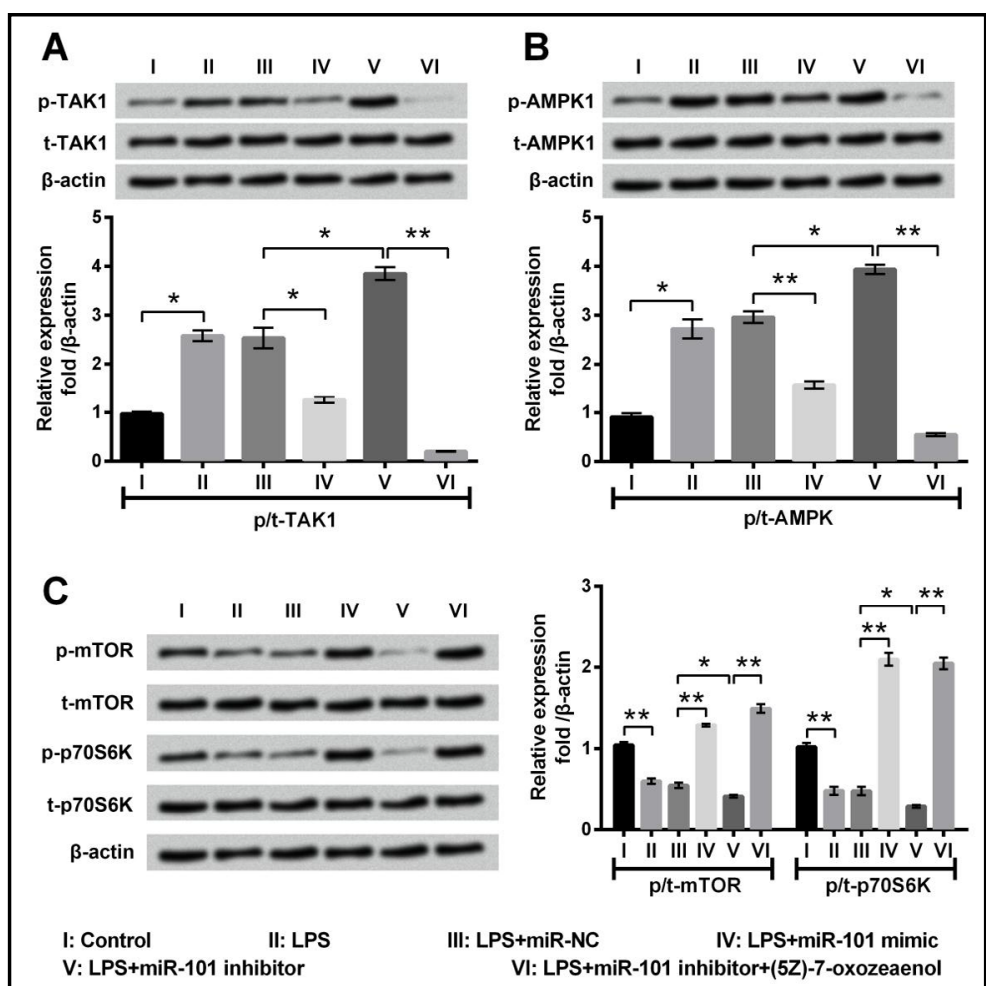

V: LPS+miR-101 inhibitor

IV: LPS+miR-101 mimic VI: LPS+miR-101 inhibitor+(5Z)-7-oxozeaenol 


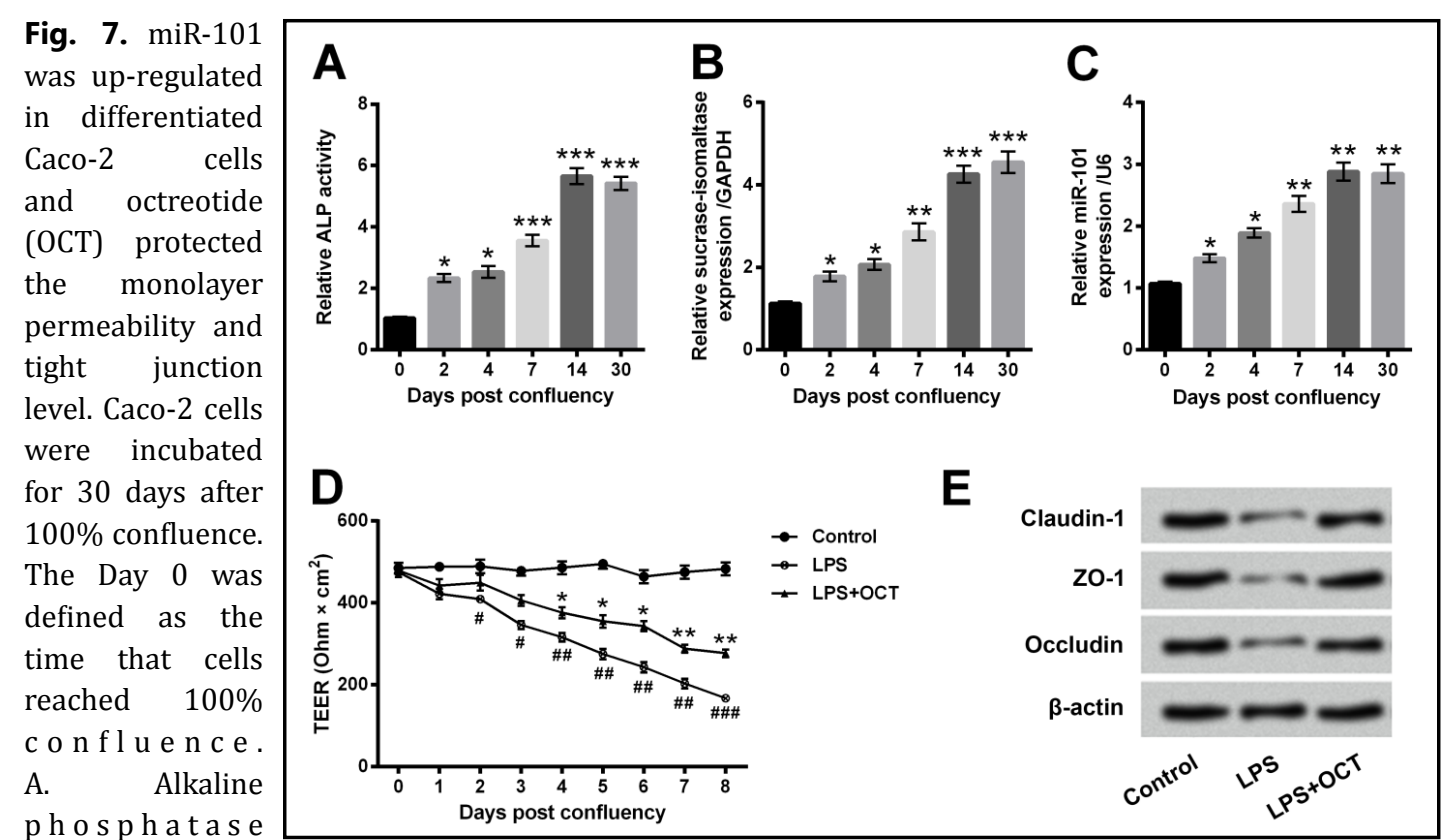

(ALP) activity

assay. Expression of sucrase-isomaltase mRNA (B) and miR-101 (C) was determined by quantitative reverse transcription PCR. Caco-2 cells were incubated for 8 days after 100\% confluence. D. Measurements of transepithelial electrical resistance (TEER). E. Expression of tight junction proteins by Western blot analysis. Data are presented as the mean \pm SEM of three independent experiments. In A-C, * indicates significant difference compared with the Day 0 group. *, $\mathrm{P}<0.05$; ${ }^{* *}, \mathrm{P}<0.01 ;{ }^{* * *}, \mathrm{P}<0.001$. In $\mathrm{D}$, * indicates significant difference compared with the LPS group. ${ }^{*}, \mathrm{P}<0.05$; ${ }^{* *}, \mathrm{P}<0.01$. \# indicates significant difference compared with the control group. \#, $\mathrm{P}<0.05$; \#\#, $\mathrm{P}<0.01$; \#\#\#, $\mathrm{P}<0.001$. LPS, lipopolysaccharide.

$P<0.001$ ) indicated that cell differentiation was induced successfully. Results in Fig. 7C showed that miR-101 level was significantly up-regulated in differentiated Caco-2 cells $(P<$ 0.05 or $P<0.01$ ). Moreover, TEER as well as expression of claudin-1, Z0-1 and occludin was significantly decreased by LPS $(P<0.05, P<0.01$ or $P<0.001)$, whereas the decreases were mitigated by OCT pre-treatments $(P<0.05$ or $P<0.01)$. Results illustrated that miR-101 was up-regulated in differentiated Caco-2 cells and OCT protected the monolayer permeability and tight junction level.

\section{Discussion}

Although OCT can alleviate intestinal mucositis which is induced by anticancer therapy, detailed molecular mechanisms remain unclear. On the other hand, patients are commonly received OCT by continuous intravenous injection in clinic due to the low enteral absorption of OCT after oral administration. Therefore, regulatory mechanisms of OCT are urgently needed to improve prognosis of therapy as well as exploration of innovative therapeutic targets for intestinal mucositis. LPS is a virulent component from gram-negative bacteria that can induce excessive immune response through TLR4 [25]. Many TLRs including TLR2, TLR4, TLR5 and TLR9 are involved in intestinal mucositis [26]. Taken into consideration that LPS can give rise to tight junction dysfunction and thereby induces mucosal hyperpermeability, we used LPS-induced Caco-2 cells to mimic in vitro intestinal mucosistis. The marked decrease of cell viability after LPS treatments suggested that cell model was successfully constructed.

During autophagy, cytosolic form of LC3 (LC3-I) is converted to membrane-bound form (LC3-II) [27]. Beclin-1 is a crucial component of the autophagosome nucleation complex and is essential for initiating of autophagy [28]. As the selective cargo receptor for misfolded 


\section{Cellular Physiology Cell Physiol Biochem 2018;49:1352-1363 \\ and Biochemistry Published online: 12 September, 2018 \begin{tabular}{l|l} 
DOI: 10.1159/000493413 2018 The Author(s). Published by S. Karger AG, Basel \\
www.karger.com/cpb
\end{tabular}}

Li et al.: Role of Octreotide in Caco-2 Cells

proteins, p62 is degraded during autophagy acting as a specific substrate [29]. Therefore, in our study, the LPS-induced increase of LC3B-II and Beclin-1 as well as down-regulation of p62 illustrated that LPS promoted autophagy. Under metabolic stress, AMPK is activated and switches on catabolic pathways to maintain the homeostasis of cellular energy [30]. Autophagy is a major catabolic pathway and it has been reported that AMPK promotes autophagy [31]. mTOR is a serine/threonine kinase that participates in regulation of cell growth, proliferation and autophagy [32]. Xiao et al. have reported that mTOR activation reflects inhibited autophagy [33]. Consistent with those previous studies, we proved that LPS might induce autophagy through activating AMPK and inhibiting the mTOR pathway.

We next explored the effects of OCT on LPS-treated Caco-2 cells. After stimulation with OCT, we found OCT improved cell viability and repressed autophagy through inhibiting AMPK and activating the mTOR pathway in LPS-treated Caco-2 cells. Baf-A1, an inhibitor of autophagosome-lysosome fusion, is widely used for determination of autophagic flux in vitro [34]. In our study, we also introduced Baf-A1 to explore how OCT affected autophagy. During autophagy, the autophagosome-lysosome fusion is followed by the degradation of autophagosome. Expression of LC3B-II in the Baf-A1 group and the Baf-A1 + OCT group was unchanged, illustrating that there is no significant effect of OCT on autophagosome formation, since expression of LC3B-II was stable when the degradation of autophagosome was inhibited. However, expression of LC3B-II in the OCT group was observably higher than the Control group, illustrating that OCT repressed degradation of autophagosome. No significant difference of LC3B-II was observed between the OCT group and the Baf-A1 + OCT group, verifying that OCT could repress degradation of autophagosome.

A previous study has shown that sodium butyrate exerts an anti-proliferative effect on Caco-2 cells through regulation of miR-203 [35]. Yu et al. have also proven that miR-217 acts as a downstream effector of long non-coding RNA CRNDE in Caco-2 cells [36]. In addition, mounting evidence has revealed the inhibitory effects of miR-101 on autophagy in tumor cells $[15,16]$. Considering the effects of OCT on autophagy, we hypothesized that miR101 might be a downstream effector of OCT and further explored the potential interaction between miR-101 and OCT treatments in LPS-treated Caco- 2 cells. Accordingly, in LPStreated cells, miR-101 level was elevated by OCT treatments. In addition, the effects of OCT on LPS-induced autophagy were reversed when the miR-101 was knocked down, indicating that OCT reduced autophagy through up-regulating miR-101 expression.

Accumulating evidence has reported that TAK1 regulates phosphorylation of AMPK, and thereby represses the mTOR complex 1, leading to increase of autophagy [17]. p70S6K is a direct substrate of mTOR, and can be phosphorylated by mTOR. Another literature also stated that TAK1 binds to p70S6K, resulting in suppression of p70S6K phosphorylation [37]. Therefore, TAK1 is a crucial factor in the LPS-induced autophagy of Caco-2 cells. Since OCT repressed LPS-induced autophagy in Caco-2 cells through up-regulating miR-101, we explored the possible interaction between miR-101 and TAK1 in LPS-treated Caco- 2 cells. In our study, we found that miR-101 could inhibit TAK1 activation, and miR-101 inhibitioninduced increase of autophagy was significantly reduced when the TAK1 phosphorylation was suppressed. That is to say, miR-101 might repress autophagy through blocking TAK1 activation in LPS-treated Caco-2 cells.

The role of miR-101 and OCT in the differentiation state of Caco- 2 cells was studied to reflect the situation that occurred in vivo. ALP activity and expression of sucrase-isomaltase are two differentiation markers [38]. Enhancements of these two factors after cell culture over a period of 30 days confirmed that Caco- 2 cell differentiation was induced. We found miR-101 expression level was significantly up-regulated in differentiated cells, verifying the significant function of miR-101. Effects of OCT on monolayer permeability and tight junction level were explored through measurements of TEER and tight junction proteins. Sheth et al. have shown that LPS causes an acute drop in TEER and down-regulation of occludin and Z0-1 [39]. Consistently, in our study, TEER and expression of tight junction proteins were reduced by LPS, illustrating that LPS destroyed monolayer permeability and tight junction level. We also found OCT could protect monolayer permeability and tight junction level 


\section{Cellular Physiology Cell Physiol Biochem 2018;49:1352-1363 \begin{tabular}{l|l} 
DOI: 10.1159/000493413 & $\begin{array}{l}\text { O 2018 The Author(s). Published by S. Karger AG, Basel } \\
\text { www.karger.com/cpb }\end{array}$ \\
\hline
\end{tabular}

which were associated with intestinal mucositis in LPS-treated Caco-2 cells, suggesting a potential application of OCT in treatment of intestinal mucositis.

The interaction among miR-101, mTOR and TAK1 may be affected by other cells present in the intestinal tract, including dendritic cells and other factors that are capable of modulating autophagy and retention of the epithelial functions. Therefore, a 3D in vitro model will offer the possibility of a benchmark much more close to the real organ. The regulatory mechanism of OCT involved in miR-101 and TAK1 is waiting to be proven in a 3D in vitro model in the future.

\section{Conclusion}

We preliminarily studied the role of OCT in autophagy of Caco-2 cells as well as the underlying mechanisms. Accordingly, autophagy could be attenuated by OCT through miR101-mediated inactivation of TAK1, along with inactivation of AMPK and activation of the mTOR pathway in LPS-treated Caco-2 cells. Our studies lay a theoretical foundation for the regulatory mechanism of OCT in autophagy of Caco-2 cells, which may assist in exploration of innovative therapeutic targets for intestinal mucositis.

\section{Acknowledgements}

This work was supported by National Nature Science Foundation of China (No. 81500587), and Natural Science Foundation of Shandong Province (No. BS2015SW021 and No.ZR2018PH005), and Science and Technology Project of Yantai (No. 2016ZH084).

\section{Disclosure Statement}

There is no conflict of interest.

\section{References}

-1 Sonis ST, Elting LS, Keefe D, Peterson DE, Schubert M, Hauer-Jensen M, Bekele BN, Raber-Durlacher J, Donnelly JP, Rubenstein EB: Perspectives on cancer therapy-induced mucosal injury: pathogenesis, measurement, epidemiology, and consequences for patients. Cancer 2004;100:1995-2025.

-2 Sano T, Utsumi D, Amagase K, Matsumoto K, Tominaga M, Higuchi K, Takeuchi T, Kato S: Lafutidine, a histamine $\mathrm{H} 2$ receptor antagonist with mucosal protective properties, attenuates 5 -fluorouracil-induced intestinal mucositis in mice through activation of extrinsic primary afferent neurons. J Physiol Pharmacol 2017;68:79-90.

-3 Prisciandaro LD, Geier MS, Butler RN, Cummins AG, Howarth GS: Evidence Supporting the use of Probiotics for the Prevention and Treatment of Chemotherapy-Induced Intestinal Mucositis. Crit. Rev. Food Sci 2011;51:239-247.

-4 Sonzini S, McCune JA, Ravn P, Scherman OA, van der Walle CF: A simple supramolecular assay for drug detection in urine. Chem Commun (Camb) 2017;53:8842-8845.

$\checkmark 5$ Giustina A, Chanson P, Kleinberg D, Bronstein MD, Clemmons DR, Klibanski A, van der Lely AJ, Strasburger CJ, Lamberts SW, Ho KKY, Casanueva FF, Melmed S: Expert consensus document: A consensus on the medical treatment of acromegaly. Nat Rev Endocrinol 2014;10:243.

-6 Kulke MH: Somatostatin Analogues in Neuroendocrine Tumors. J Natl Compr Canc Netw 2016;14:241-242.

7 Zhou DX, Zhou HB, Wang Q, Zou SS, Wang H, Hu HP: The Effectiveness of the Treatment of Octreotide on Chylous Ascites After Liver Cirrhosis. Digest Dis Sci 2009;54:1783-1788.

-8 Peterson DE, Bensadoun RJ, Roila F: Management of oral and gastrointestinal mucositis: ESMO clinical recommendations. Ann Oncol 2008;19:ii122-125. 


\section{Cellular Physiology Cell Physiol Biochem 2018;49:1352-1363 and Biochemistry \begin{tabular}{l|l} 
DOI: 10.1159/000493413 & $\begin{array}{l}\text { C } 2018 \text { The Author(s). Published by S. Karger AG, Basel } \\
\text { www.karger.com/cpb }\end{array}$
\end{tabular}

9 DeMeo MT, Mutlu EA, Keshavarzian A, Tobin MC: Intestinal permeation and gastrointestinal disease. J Clin Gastroenterol 2002;34:385-396.

10 Li X, Wang Q, Xu H, Tao L, Lu J, Cai L, Wang C: Somatostatin regulates tight junction proteins expression in colitis mice. Int J Clin Exp Pathol 2014;7:2153-2162.

11 Mizushima N: PL-02Monitoring of autophagosome formation and maturation. Microscopy 2016;65:i3-i3.

12 Wan SX, Shi B, Lou XL, Liu JQ Ma GG, Liang DY, Ma S: Ghrelin protects small intestinal epithelium against sepsis-induced injury by enhancing the autophagy of intestinal epithelial cells. Biomed Pharmacother 2016;83:1315-1320.

13 Comincini S, Allavena G, Palumbo S, Morini M, Durando F, Angeletti F, Pirtoli L, Miracco C: microRNA-17 regulates the expression of ATG7 and modulates the autophagy process, improving the sensitivity to temozolomide and low-dose ionizing radiation treatments in human glioblastoma cells. Cancer Biol Ther 2013;14:574-586.

14 Zhu H, Wu H, Liu X, Li B, Chen Y, Ren X, Liu CG, Yang JM: Regulation of autophagy by a beclin 1-targeted microRNA, miR-30a, in cancer cells. Autophagy 2009;5:816-823.

15 Xu L, Beckebaum S, Iacob S, Wu G, Kaiser GM, Radtke A, Liu C, Kabar I, Schmidt HH, Zhang X, Lu M, Cicinnati VR: MicroRNA-101 inhibits human hepatocellular carcinoma progression through EZH2 downregulation and increased cytostatic drug sensitivity. J Hepatol 2014;60:590-598.

16 Guo J, Huang X, Wang H, Yang H: Celastrol Induces Autophagy by Targeting AR/miR-101 in Prostate Cancer Cells. Plos One 2015;10:e0140745.

17 Inokuchi-Shimizu S, Park EJ, Roh YS, Yang L, Zhang B, Song J, Liang S, Pimienta M, Taniguchi K, Wu X, Asahina K, Lagakos W, Mackey MR, Akira S, Ellisman MH, Sears DD, Olefsky JM, Karin M, Brenner DA, et al.: TAK1-mediated autophagy and fatty acid oxidation prevent hepatosteatosis and tumorigenesis. J Clin Invest 2014;124:3566-3578.

-18 Gao J, Gao J, Qian L, Wang X, Wu M, Zhang Y, Ye H, Zhu S, Yu Y, Han W: Activation of p38-MAPK by CXCL4/ CXCR3 axis contributes to p53-dependent intestinal apoptosis initiated by 5-fluorouracil. Cancer Biol Ther 2014;15:982-991.

19 Mayo BJ, Stringer AM, Bowen JM, Bateman EH, Keefe DM: Irinotecan-induced mucositis: the interactions and potential role of GLP-2 analogues. Cancer Chemother Pharmacol 2017;79:233-249.

20 Guo S, Al-Sadi R, Said HM, Ma TY: Lipopolysaccharide causes an increase in intestinal tight junction permeability in vitro and in vivo by inducing enterocyte membrane expression and localization of TLR-4 and CD14. Am J Pathol 2013;182:375-387.

21 Huang Y, Li N, Liboni K, Neu J: Glutamine decreases lipopolysaccharide-induced IL-8 production in Caco-2 cells through a non-NF- $\kappa$ B p50 mechanism. Cytokine 2003;22:77-83.

22 Li Y, Duan Z, Tian Y, Liu Z, Wang Q: A Novel Perspective and Approach to Intestinal Octreotide Absorption: Sinomenine-Mediated Reversible Tight Junction Opening and Its Molecular Mechanism. Int J Mol Sci 2013;14:12873.

23 Araujo JR, Correia-Branco A, Ramalho C, Keating E, Martel F: Gestational diabetes mellitus decreases placental uptake of long-chain polyunsaturated fatty acids: involvement of long-chain acyl-CoA synthetase. J Nutr Biochem 2013;24:1741-1750.

-24 Livak KJ, Schmittgen TD: Analysis of relative gene expression data using real-time quantitative PCR and the 2(-Delta Delta C(T)) Method. Methods 2001;25:402-408.

25 Shigemori S, Namai F, Yamamoto Y, Nigar S, Sato T, Ogita T, Shimosato T: Genetically modified Lactococcus lactis producing a green fluorescent protein-bovine lactoferrin fusion protein suppresses proinflammatory cytokine expression in lipopolysaccharide-stimulated RAW 264.7 cells. J Dairy Sci 2017;100:7007-7015.

-26 Stringer A: Interaction between Host Cells and Microbes in Chemotherapy-Induced Mucositis. Nutrients 2013;5:1488.

27 Tanida I, Ueno T, Uchiyama Y: Chapter Six - Use of pHlurorin-mKate2-human LC3 to Monitor Autophagic Responses; in (Galluzzi L, Bravo-San Pedro JM, and Kroemer G, eds) Methods in Enzymology. Methods Enzymol 2017;587:87-96.

28 Zhang C, Jia X, Wang K, Bao J, Li P, Chen M, Wan J-B, Su H, Mei Z, He C: Polyphyllin VII Induces an Autophagic Cell Death by Activation of the JNK Pathway and Inhibition of PI3K/AKT/mTOR Pathway in HepG2 Cells. Plos One 2016;11:e0147405. 


\section{Cellular Physiology Cell Physiol Biochem 2018;49:1352-1363 \begin{tabular}{l|l|l} 
and: 10.1159/000493413 & $\begin{array}{l}\text { O 2018 The Author(s). Published by S. Karger AG, Basel } \\
\text { www.karger.com/cpb }\end{array}$ \\
\hline
\end{tabular}

29 Sun S, Chen J, Yan Q, Bao C, Fu Q: Autophagy-related protein p62 expression is associated with clinicopathologic features and prednisone plus ctx induction treatment efficacy in lupus nephritis. Lupus Sci Med 2017;4:A143-A143.

-30 Hardie DG: AMPK: positive and negative regulation, and its role in whole-body energy homeostasis. Curr Opin Cell Biol 2015;33:1-7.

-31 Zhang CS, Lin SC: AMPK Promotes Autophagy by Facilitating Mitochondrial Fission. Cell Metabolism 2016;23:399-401.

-32 Kim YC, Guan KL: mTOR: a pharmacologic target for autophagy regulation. J Clin Invest 2015;125:25-32.

-33 Xiao J, Zhu X, Kang B, Xu J, Wu L, Hong J, Zhang Y, Ni X, Wang Z: Hydrogen Sulfide Attenuates Myocardial Hypoxia-Reoxygenation Injury by Inhibiting Autophagy via mTOR Activation. Cell Physiol Biochem 2015;37:2444-2453.

-34 Mauvezin C, Neufeld TP: Bafilomycin A1 disrupts autophagic flux by inhibiting both V-ATPase-dependent acidification and Ca-P60A/SERCA-dependent autophagosome-lysosome fusion. Autophagy 2015;11:14371438.

-35 Han R, Sun Q Wu J, Zheng P, Zhao G: Sodium Butyrate Upregulates miR-203 Expression to Exert AntiProliferation Effect on Colorectal Cancer Cells. Cell Physiol Biochem 2016;39:1919-1929.

-36 Yu B, Ye X, Du Q, Zhu B, Zhai Q Li XX: The Long Non-Coding RNA CRNDE Promotes Colorectal Carcinoma Progression by Competitively Binding miR-217 with TCF7L2 and Enhancing the Wnt/beta-Catenin Signaling Pathway. Cell Physiol Biochem 2017;41:2489-2502.

-37 Shin JH, Min SH, Kim SJ, Kim YI, Park J, Lee HK, Yoo OJ: TAK1 regulates autophagic cell death by suppressing the phosphorylation of p70 S6 kinase 1. Sci Rep 2013;3:1561.

-38 Astarci E, Erson-Bensan AE, Banerjee S: Matrix metalloprotease 16 expression is downregulated by microRNA-146a in spontaneously differentiating Caco-2 cells. Dev Growth Differ 2012;54:216-226.

-39 Sheth P, Delos Santos N, Seth A, LaRusso NF, Rao RK: Lipopolysaccharide disrupts tight junctions in cholangiocyte monolayers by a c-Src-, TLR4-, and LBP-dependent mechanism. Am J Physiol Gastrointest Liver Physiol 2007;293:G308-318. 